\title{
Diseñar actos pedagógicos exitosos con adolescentes escolarizados(as) a partir de la incertidumbre: una aplicación de la pedagogía performativa intercultural en una Secundaria en Medellín, Colombia
}

Anja María MacKeldey, Universidad de Antioquia Sede Medellín/Colegio Alemán Medellín, Colombia

\begin{abstract}
Resumen: El desmoronamiento de estructuras supuestamente simbólicas para la eternidad como dios, familia, nación, sexo o género, desata en los y las ciudadanos(as) de los mundos una determinada incertidumbre que influye en nuestro pensar y actuar. Semejantes desmoronamientos de nuestras creencias son considerados por la pedagogía performativa intercultural (PPI) una oportunidad de percibirnos y/o diseñarnos como personas tradicional y estructuralmente actuadas, y al mismo tiempo como personas actuantes en nuestros juegos identitarios, por lo que convoca a realizar actos pedagógicos acordes a este enfoque quiasmático. En este contexto, la PPI asume los mundos de vida adolescentes y su pensamiento randómico como manifestaciones paradigmáticas de la incertidumbre. Técnicas mayéutico-didácticas constructivas para poner en escena y aprender a leer el tanto...como... quiasmático propuesto por la PPI, demostraron ser la autoetnografía, el juego dramático, el método documental y la retroalimentación. Finalmente, este aporte busca evidenciar por medio de una proyección esquemática de la PPI y unas experiencias ejemplares (2005-2013) que el aprovechamiento de la incertidumbre facilita procesos de enseñanza y aprendizaje exitosos con adolescentes esolarizados(as). Con eso, ponemos en discusión la PPI como dispositivo para una práctica pedagógica co-constructiva en materias que se relacionan directa o transversalmente con la educación democrática.
\end{abstract}

Palabras clave: procesos educativos exitosos, incertidumbre, pedagogía performativa intercultural, educación democrática

\begin{abstract}
The decline of supposedly symbolic structures devised for eternity, such as God, family, nation, sex or gender, unties in the citizens of the worlds a certain uncertainty that influences our thinking and acting. Intercultural Performative Pedagogy (IPP) considers such declines of our beliefs to be an opportunity for us to perceive and/or devise ourselves as actuated subjects that follow traditional and structural patterns and, at the time, as subjects acting within our own identity games. For that reason, it calls for pedagogical actions that are consistent with this chiasmatic focus. In this context, IPP sees adolescent life worlds and its random thinking as paradigmatic manifestations of uncertainty. Constructive maieutic didactic techniques designed to put on stage and learn how to read the chiasmatic MacKeldey this as well as that" as suggested by IPP have proved to be auto-ethnography, the dramatic play, the documentary methodology and feedback. In conclusion, what this writing seeks to prove by pointing to chiasmatic IPP and a few exemplary experiences collected between 2005 and 2014 is that taking advantage of uncertainty eases successful teaching and learning processes with school adolescents. This way, we consider IPP to be a mechanism that is suitable for co-constructive pedagogical practice in subjects that relate to democratic education in a direct or transversal manner.
\end{abstract}

Keywords: Successful Educational Processes, Uncertainty, Intercultural Performative Pedagogy, Democratic Education

\section{Incertidumbre y educación}

La necesidad de incluir en los actos y reflexiones pedagógicas la incertidumbre que resulta de la disolución de estructuras y creencias inamovibles como la familia, la religión, la nación, el sexo o género, no es apenas un fenómeno del siglo XXI: las primeras advertencias al respecto provienen del final del siglo XX. Morin (1999) considera, por ejemplo, necesario que los procesos educativos enfrenten la incertidumbre inherente a los conocimientos cuyo valor a largo plazo no puede ser asegurado. El autor llama incluso "la enfermedad de nuestra mente" (p. 46) a la ansiedad humana de

Revista Internacional de Educación y Aprendizaje

Volumen 3, Número $2<$ http://sobrelaeducacion.com/>, ISSN 2255-453X

(C) Global Knowledge Academics. Anja María MacKeldey

Todos los derechos reservados. Permisos: soporte@gkacademics.com 
querer aniquilar las incertidumbres que nos sucedan. Si podemos afirmar que en el transcurso del siglo XXI llegan a ser cada vez más virulentas las manifestaciones de incertidumbres provocadas por la aceleración de las transformaciones cada vez más tajantes como las de los mundos virtuales y sus dispositivos o las transformaciones políticas como la instalación del sistema de la nueva gobernanza (New Governance).

En este contexto, no asombra la posición del pedagogo español Escolet (Borrajo, 2012) cuando afirma que la educación para la incertidumbre es una prioridad en nuestros tiempos, por lo que los y las estudiantes deben aprender a pensarse a sí mismos, también con la ayuda de los afectos. Añade que esta exigencia no puede hacerse realidad en el sistema educativo español mientras éste se siga destacando por su rigidez y énfasis en aprendizajes cognitivos.

El peligro de una escuela rígida en la que finalmente se enseña mucho y se aprende poco, fue percibido ya por Comenio, pero sigue siendo una realidad muy común en los sistemas educativos, que se refleja en el alto porcentaje de deserción estudiantil en muchos países de América Latina, como Colombia donde desertaron en 2013más de cuatrocientos mil estudiantes de sus instituciones educativas básicas y casi la mitad de los estudiantes universitarios (Semana, 2013;Olave-Arias, G.; Rojas-García, I.; Cisneros-Estupiñán, M., 2013).Las causas pueden encontrarse en las problemáticas virulentas del sistema educativo estatal, falta de inversión suficiente, problemas de orden público e incertidumbres para el futuro de las nuevas generaciones. Al mismo tiempo, se perciben determinados giros necesarios hacia un aprendizaje más significativo como la introducción dela "lectura crítica" a los exámenes de Estado en la educación media (llamados Pruebas Saber 11) y que reúnen bajo este concepto dos materias: lengua española y filosofía. Con ello se pretende incluir a los y las estudiantes más como actores del conocimiento. El Estado colombiano logra en este aspecto superar una educación orientada, en palabras de Freire (1973), al "concepto banquero" que "con su tendencia a porcionar todo" (p. 85) convierte personas en objetos y es, por ende "necrófilo". Pero en general siguen existiendo muchos rituales monótonos en las aulas de clase que contribuyen a que muchos estudiantes se vuelvan insensibles a los procesos de enseñanza y aprendizaje, por lo cual se convierten con frecuencia en cuasi rumiantes de hojas de trabajo con respuestas prefabricadas.

Si consideramos la educación en el nivel global en Occidente, las pruebas PISA pretenden "estandarizar" cada vez más los procesos y resultados (y con ello, los sujetos) en el campo educativo. Brinkmann (2009) observa en la creciente estandarización de la educación una creciente estandarización formativa, en la que se insertan los Colegios Alemanes en el Exterior (entre los cuales se halla nuestro contexto educativo), por medio de un "certificado de calidad" sin el cual, pierde su derecho a ser un "Colegio Alemán" y recibir los respectivos recursos financieros. Manifestaciones de estándares de calidad como el certificado mencionado que se renueva cada cuatro años, traen consigo la legitimización de una sociedad de control cuando supervisan de forma regular a estudiantes y profesores con base en exámenes y observaciones de clase orientados en el output y realizados bajo una presión temporal cada vez más intensa.

\section{Condiciones necesarias para una fusión co-constructiva de la educación y las incertidumbres}

\section{Necesidades organizacionales}

Una condición previa organizacional para poder transformar constructivamente las realidades descritas, consiste en que no solo profesores y profesoras saquen provecho de los estados de incertidumbre, sino que también los directivos, las comunidades profesionales de aprendizaje e incluso los ministerios de educación entiendan y/o permitan que se tome ventaja de la incertidumbre inherente a los procesos educativos y a las propiedades competitivas de la estandarización educativa. Con eso, las personas encargadas pueden incentivar procesos exitosos y contribuir a una superación de los miedos (creados) en una comunidad educativa que teme, o salir de las conceptualizaciones memo-

\footnotetext{
${ }^{1}$ La autora del artículo figura como traductora de todos los textos en alemán o inglés al español.
} 
rísticas de la educación o quedarse entre los frustrados que no responden de la manera estandarizada prevista. En 2013, por ejemplo, Colombia obtuvo en las pruebas internacionales de la medición de la calidad educativa PISA el puesto 61 entre 65 países participantes y debe repensar especialmente la educación pública más allá de la transformación prometedora de la "lectura crítica" en las pruebas Saber 11. La misma prueba provocó a comienzos del siglo XXI (2001) el llamado "PISASchock" en Alemania, cuando ese país obtuvo muy malos resultados, por ejemplo en la competencia lectora y puso en marcha un gran número de reformas educativas, como el cuestionamiento del sistema educativo tripartito y excluyente.

Las personas y/o autoridades competentes deben animar y formar a todos los miembros de la comunidad educativa - empezando con los y las profesores(as) y estudiantes - a pensar por sí mismos, quitar el miedo a situaciones imprevistas y revocar el carácter lúdico del aprendizaje con sus propiedades contingentes. Esto último, se puede lograr especialmente mediante proyectos de vida de los estudiantes en los que "aprendan a jugar", a ser simultáneamente niño(a), adolescente y adulto(a) en el nivel cognitivo, social y corporal de una forma randómica, sin que el entorno interprete esta actitud de una forma binómica: "correcto"-“incorrecto" o incluso como "verdadero"-"falso". Es decir, sin reconocer otros matices posibles entre los polos.

\section{Necesidades conceptuales}

Para poder co-construir la formación de sujetos que se (auto)entiendan y representen como actores actuados conscientes de sus múltiples identidades simultáneas, los procesos de enseñanza y aprendizaje deben basarse en la comprensión de que en los actos educativos oscilan constantemente nuestros papeles de ser estudiantes y/o educadores.

En este contexto, la Pedagogía Performativa Intercultural (PPI) pretende conectar estudios educativos y propuestas pedagógicas existentes que contribuyen a la percepción de esta horizontalidad contingente de los procesos de enseñanza y aprendizaje, y proponer jugar con las posibilidades que se perfilan entre los innumerables polos que se desdibujan en este nuevo rizoma llamado PPI.

Figura 1: A Fresh Rhizome of Cimicifuga Racemosa (Natural Size)

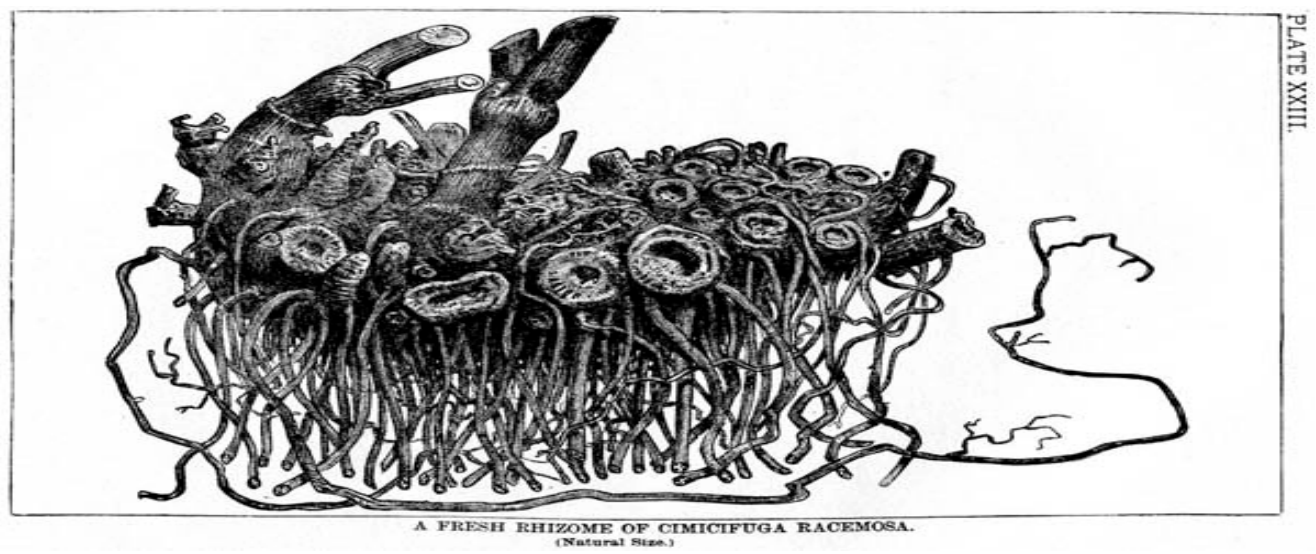

Fuente(s): http://www.urbagram.net/v1/show/Microplexes, s.f.

En lo siguiente mencionaremos sólo unos pocos de los más importantes antecedentes de la PPI. En 1997,ParraSandoval constató en el primer tomo de Atlántida, la cultura fragmentada-un proyecto que dejó hablar a un sinnúmero de adolescentes colombianos urbanos-,que la simultaneidad de la lentitud de las prácticas escolares, la fugacidad del mundo moderno occidental y sus tecnologías, y la convivencia del tiempo premoderno, moderno y posmoderno en la sociedad colombiana llevan a que "[u]na respuesta única, homogénea, surgida de una personalidad centrada, no fragmentada, crearía conflictos dolorosos" (p. 157).Además, los y las jóvenes dentro de las ciudades moderniza- 
das experimentan lo que Parra Sandoval (1997) denomina un presente expandido, que pone en un segundo lugar el futuro y el pasado, aunque sea al mismo tiempo un presente frágil y fragmentado por su inmensa velocidad.

Otro concepto que abarca la incertidumbre en las vidas de los y las adolescentes es el pensamiento randómico. Este se desarrolla en el estudio titulado Design: designar/diseñar el cuerpo joven urbano (2003), realizado con adolescentes de clase media alta y alta en la ciudad colombiana de Cali y coordinado por Rocío del Socorro Gómez. Los y las autores(as) definen el pensamiento randómico como una

manera de pensar por asociaciones concurrentes, por lateralidades, saltándose secuencias y consecuencias, este modo "no-lineal" de articular ideas-escenas, este pensamiento fuertemente visual, es consistente con el mundo real que lo origina, un mundo que no se atiene a conductas heredadas y secuenciadas para producir y crear (p. 103).

Este concepto no-lineal repercute en la PPI, cuando ésta parte de la constitución de sujetos compuestos por muchas "identidades" simultáneas y hace resaltar el que las personas entre 13 y 18 años o los llamados adolescentes, no se empeñen "en la búsqueda de su identidad", sino que están poniendo a prueba una gran variedad de identidades posibles en sus diferentes contextos familiares, escolares, de pares, etc. Se trata más bien de un playing identity con todas las libertades y restricciones que ofrece el mundo del juego y estas oscilaciones identitarias no deberían ser entendidas como mentiras por el mundo de los adultos. Tampoco debemos entender estos juegos identitarios como juegos inconscientes e incesantes, sino que debemos asumir con la PPI que los sujetos optamos cada cuanto por una identidad estratégica que emerge, según Hall (2003), en la sutura entre los discursos prácticos y los procesos subjetivantes y equivale siempre a un posicionamiento consciente. Optar por cierta identidad posicional y fragmentada, surge como una respuesta de resistencia por parte de los sujetos contra el riesgo de convertirnos en unos yoes incesantemente performativos, es decir yoes o identidades constantemente modificantes y modificados.

Por último, tenemos que mencionar el pilar que aparece como actor y actuado en nuestras puestas en escena identitarias: nuestro cuerpo. En una comunidad educativa por ejemplo, este dar y darse una forma se materializa en la interrelación de los cuerpos estudiantiles y profesorales: tanto los y las profesores(as) transportamos y transmitimos con nuestras historias de vida un determinado currículo oculto, como los y las estudiantes transportan y transmiten sus historias de vida. Ambas partes co-construimos el éxito y/o fracaso de los procesos formativos y educativos, a partir de las respectivas escenificaciones de nuestros antecedentes. De ahí la necesidad de incluir la autoetnografía como materia autorreflexiva en la formación de maestros y maestras. Además, debemos comprender qué saberes actúan sobre nuestros cuerpos para prevenir la situación extrema de alienación que pueden experimentar personas en situaciones extremas y de opresión que se (auto)perciben como meramente actuadas, una condición humana evocada por Garzón Martínez (2007) en su pregunta "¿qué pasa con aquellas para quienes gritar no es una opción, pues su voz ha sido secuestrada por discursos médicos, blancos y patriarcales que hablan por ellas?” (p. 164).

Para poder manejar los saberes reconocidos debemos liberarnos de fuerzas tradicionalmente opresoras, también de la (auto)atribución de un papel pasivo y de víctima, en cuanto invocamos la incertidumbre identitaria y reconocemos en el sector educativo a los y las estudiantes y profesores simultáneamente como actuados y actores. Con eso podemos hacer valer por ejemplo la actitud de resiliencia que cada vez más personas en situaciones extremamente difíciles y de opresión están oponiendo a las situaciones adversas y sus victimarios, y al mismo tiempo la desarrollan a favor de su deseo de obtener un papel constructivo como actor autónomo de su propia vida. (cfr. SARAS "South American Institute for Resilience and Sustainability Studies en www.saras-institute.org).

Cuando la educación se apropia de su paradoja principal formulada por Zirfas (2001): "la intencionalidad pedagógica no funciona desde un principio: No podemos querer la formación del otro" (p. 86) y se comprende que "en la educación y la formación se trata, por ende, de "educatio", de conducir hacia fuera de supuestas certezas para poder valorar debidamente la singularidad del otro en el 
proceso pedagógico" (p. 87), entonces es la tarea de la educación hacer comprensibles y manejables estas identidades oscilantes entre ser actores y actuados, y por ende, cargados de incertidumbres.

La toma de conciencia en el acto educativo de tener que "conducir hacia fuera de supuestas certezas" puede volverse sólo realidad si hacemos tangible el momento y/o el tiempo-espacio en cuanto ponemos en escena actos de enseñanza y aprendizaje que consideran las relaciones rizomáticas de las singularidades.

\section{La pedagogía performativa intercultural como ayuda para moldear la incertidumbre}

\section{Accesos al tiempo-espacio de la incertidumbre}

En el primer momento nos acercamos a lo que Buber (1982, cit. en Muth, s.f.) conceptualiza como la "esfera del entremedio (Dazwischen) Es una categoría primordial de la realidad humana. ... más allá de lo subjetivo, más acá de lo objetivo, en la cresta delgada en la que suceden y se encuentran el Yo y el Tú, se halla el reino del entremedio"'(p. 9).Para imaginar con más facilidad la constitución del espacio entremedio en el campo educativo, Wang (2005) define la aporía derridiana y su utilidad para la educación intercultural, como "la posibilidad de lo imposible [que] nos invita a experimentarla como tal, incluyendo sus contradicciones lógicas. La posibilidad de "llegar a pasar" y de "llegar sin dar el paso" convierte el acto de pasar el límite en una paradoja”(p. 48).

La apertura al otro no equivale en la lectura de Wang (2005) a un estado de indiferencia o una aceptación de "todo" (el llamado anything goes), sino que obedece al doble gesto de distanciarse de lo establecido e intervenir simultáneamente desde este mismo establishment. Al mismo tiempo, debemos cumplir con el doble gesto de tomar una propia decisión y estar abierto a posibles alternativas. Esta duplicidad del gesto lleva a un estado de desasosiego o incertidumbre duradero, un estado indispensable para el encuentro con el "extranjero en nosotros mismos" como propone la psicoanalista y semióloga búlgara Kristeva (1997).

El desasosiego o encuentro con el extranjero en nosotros mismos que nos estremece para percibir y manejar la incertidumbre, puede manifestarse como:"1. (temblores, explosiones): hacer temblar, sacudir 2. (fe, salud): quebrantar; (confianza) poner en duda y 3. (noticia, vivencia) conmover (profundamente), afectar, conmocionar", si damos crédito a las entradas en la página 366 para el verbo alemán erschüttern en el diccionario PONS - Schule und Studium (1999), cuyo uso es de mucho provecho para el contexto colombo-alemán que fungió como base para el desarrollo de la PPI.

Otro acercamiento a la incertidumbre en cuanto "veleidad" proviene del encuentro de blogger re:publica 2014 Into the wild en Berlín, al que asistieron aproximadamente 6.000 blogger en mayo de 2014. A este evento los organizadores convocaron con el lema "Into the wild [...] a animar, a repensar la red en la era post-Snowden, a atreverse a hacer cosas nuevas y alentar a tomar caminos de la veleidad" (Republica, 2014).Con referencia al evento, el periodista germano-austríaco Lotter (2014) subraya el valor positivo de la incertidumbre, aquí en forma de veleidad, cuando afirma que "Lo salvaje y el conocimiento son lo mismo. Ambos son veleidosos. La sociedad del conocimiento requiere valor."

Kusanowsky (2013) va incluso un paso más allá cuando cuestiona la ingenuidad humana de esperar sin pestañear que suceda una normalidad basada en repeticiones. Aplicada a la educación, esta circunstancia conlleva según el autor a que "[e]l caso extremo de todos los procesos de aprendizaje consiste en quebrar con la ingenuidad. Su condición previa debería ser, a fin de cuentas, percibirla". En cuanto que se haya efectuado la percepción de la ingenuidad y/o el quebrantamiento de la fe, el temblor o la conmoción, las y los educadores debemos tener presente con Zirfas (2001) que el trato con el otro tiene que basarse en el saber que la situación presente queda pendiente, incierta y no puede ser decidida: "La educación en este sentido es una relación con la singularidad del otro en su propiedad de no estar disponible ni sustituible." (p. 98)

En pos de efectuar un determinado acercamiento a la incertidumbre también en el nivel lingüístico, debemos considerar que los actos de habla incluyen por un lado la polisemia, es decir, las in- 
terpretaciones que diversos contextos culturales y sintagmáticos le dan a una palabra, y por otro, la greffe (injerto) derridiana que siempre es una huella de ella misma y presupone que es la palabra la que disemina los significados.

Ambas propiedades de la palabra necesitan repeticiones para que se lleve a cabo la percepción de una determinada normalidad. Consta que las repeticiones pueden devenir iteraciones, que son repeticiones modificantes de un acto (de habla) antecedente y nos empoderan a convertirnos en actores de incertidumbres.

\section{En el camino hacia la fusión exitosa de la educación con la incertidumbre}

Como ya nos hallamos en el campo de la actuación en el entremedio, debemos abordar la metodología performativa en su sentido etimológico. El concepto griego methodos significa "camino hacia", en nuestro contexto, hacia sujetos co-constructores y autorreflexivos de la incertidumbre y los juegos identitarios. En este camino, los estudiantes y los educadores en nuestros roles oscilantes debemos reconocernos como actores y actuados al mismo tiempo, y que co-construimos en este camino con toda responsabilidad y conciencia ciudadana los significados de los actos discursivos y corporales. Queremos poner énfasis en el rol de los actos corporales y/o las puestas en escena corporales porque, como dice la contraportada del libro Pädagogik des Performativen (Wulf et al., 2007): "niños, niñas y jóvenes no se educan cuando se habla sobre educación y formación sino cuando los procesos educativos y formativos se ponen en escena".

La metodología performativa cumple con esta exigencia, porque toma en cuenta en el campo educativo las puestas en escena en los siguientes ámbitos: el ámbito lingüístico donde la perfomancia aparece como contraparte a la competencia; el sector artístico, creativo e innovador (performan$c e$ ), y el ámbito socio-filosófico, desde el cual se busca entender los efectos (no el significado intencionado) de nuestros actos que pueden co-construir asimetrías y jerarquizaciones (performatividad). La metodología performativa facilita el desarrollo de las habilidades requeridas por la sociedad del conocimiento comandada por la productividad y al mismo tiempo ofrece las herramientas para contrarrestarlas. En lo siguiente se proponen unas técnicas y métodos provenientes de los campos mencionados que en torno a las incertidumbres contribuyen a llevar a una práctica exitosa las exigencias formuladas por la pedagogía performativa intercultural en cuanto a la co-construcción de sujetos autorreflexivos y conscientes de los juegos identitarios.

\section{Hablar de las incertidumbres identitarias}

Considerando los trasfondos socioeconómicos de los y las participantes, Wang (2005) recomienda que los actos educativos partan de la autocrítica en contextos con estudiantes del centro y de la autoafirmación con estudiantes marginados. La PPI añade que no podemos olvidar que una persona puede reunir identidades contradictorias a primera vista. Por ejemplo: un(a) estudiante de un colegio de clase media-alta o alta puede tener una identidad socioeconómica del centro y experimentar simultáneamente una identidad marginada por no cumplir con los estándares ideológicos o de género considerados como naturales por el contexto cultural. En el contexto colombo-alemán del Colegio Alemán Medellín, contamos con el testimonio de una estudiante futbolista de 11 er grado en 2006 a la que especialmente sus pares del mismo sexo la "molestaron y siempre lo han hecho" por su pasión al fútbol, especialmente entre el grado $3^{\circ}$ y $8^{\circ}$. Otro testimonio ofrece Luiyima de 11 er grado en 2010 , estudiante creativo, crítico y considerado como divergente a las normas instaladas por sus pares a partir del $7^{\circ}$ grado, quien dejó de compartir sus conocimientos y actitudes en el aula, porque "me cansé que no me tomen en serio, no me presten atención" (MacKeldey, 2010b).

Ambos estudiantes vivieron años de incertidumbre provocada por sus pares y fueron capaces de transformar este estado de desasosiego con ayuda de la autorreflexión. En el primer caso, la estudiante logró reconocerse en su propio ser y sólo un problema con la rodilla la alejó del futbol. En el segundo, el camino fue la autogestión, él fundó un grupo de rap en el que pudo expresarse libremente. 
Los estudiantes mencionados fueron capaces, después de muchos años de sentida exclusión, de manejar sus incertidumbres identitarias a favor de ellos mismos. Por lo menos lo exponen como tal en las entrevistas a profundidad. Esta es una técnica que propone la PPI para hacer comprensibles las incertidumbres identitarias, porque parte de la autorreflexión de los participantes y el valor de la palabra hablada o performancia. Desafortunadamente, la labor de estudiantes y educadores no permite recurrir a esta técnica muy valiosa con mucha frecuencia ya que primero, requiere mucho tiempo para acordar el lugar-tiempo ideal; segundo, para la realización y el análisis, y finalmente para la retroalimentación.

Figura 2: Un juego identitario autorreflexivo exitoso de luiyima

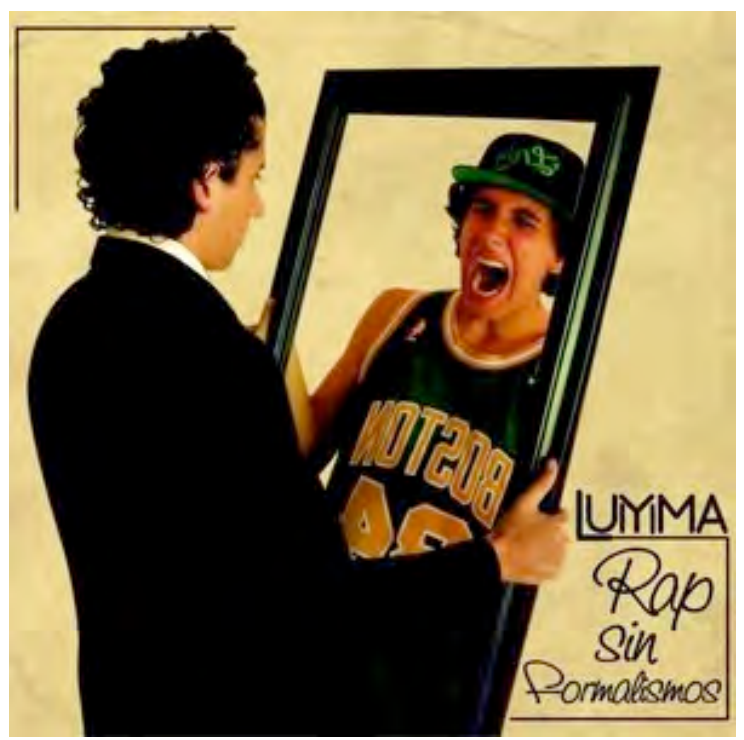

Fuente: https://a3-images.myspacecdn.com/images03/2/725aafcb514847deae1b721a61969f8c/ 300x300.jpg, s.f.

Las entrevistas con los estudiantes como todas las entrevistas a profundidad realizadas entre 2005 y 2010 en el marco del doctorado de educación, revelaron las siguientes particularidades que despertaron mucha incertidumbre: mientras que en los comienzos, la mayoría de los entrevistados mostró mucha timidez, en el transcurso de la entrevista. A medida que avanzaba la conversación, la incertidumbre también cubrió a la investigadora, probablemente porque el acto de habla obtuvo una función terapéutica, y las y los entrevistados crearon en el habla cada vez más nexos y plexos acerca de la percepción de su ser y estar de ellos mismos en el mundo.

Ofreceremos unos métodos usados en la investigación realizada y que posteriormente, se transformaron en técnicas didáctico-mayéuticas para aplicar en clase. Estas técnicas son más pragmáticas que las empleadas en la investigación, porque no exigen tanto tiempo. Además, oscilan entre su calidad de servir para la recolección de datos, por un lado, y como métodos didáctico - mayeúticos para las clases por el otro.. Empezamos con la técnica aplicada por ambos estudiantes, durante mucho tiempo de una forma no estructurada: la autorreflexión, cuyo proceso pretendemos hacer más efectivo y ordenado en forma de la autoetnografía.

\section{Escribir autorreflexivamente en forma de la autoetnografía}

Dyson (2007) propone la autoetnografía como metodología de empoderamiento para educadores y educadoras. Se trata de "una presentación desde el punto de vista de una persona, o su mapa o realidad que es construida alrededor de otras personas y por estas personas mismas" (p. 46).El 
ejercicio de la autoetnografía podría realizarse en jornadas pedagógicas con profesores y en clases de "ética" o "valores" con estudiantes, porque es una ayuda efectiva de aprender más sobre los propios actos y sus móviles para, dado el caso, modificarlos. Como ilustración, el mapa autoetnográfico que la autora elaboró en el desarrollo de la PPI en 2009. Se reproducen sólo los subtítulos de capítulos de la vida autobiográficos generales y la autofoto resultante.

Figura 3: Autofoto para autoetnografía

"Y la juventud en el ataúd"

"El mito América Latina incorporado"

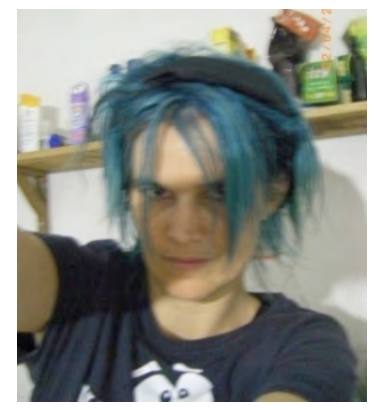

"Salud, amor y libertad"

"La gener(iz)ación de la identidad"

Fuente: MacKeldey, 2010.

\section{Considerar nuestras puestas en escena corporales y discursivas en el aula}

Butler (2002) propone combinar la tesis feminista que convoca a recuperar lo personal como asunto político, con la consideración de la categoría cuerpo, especialmente desde la tradición fenomenológica, en vez de contraponer "la mujer" a "el hombre". Para eso considera necesario superar la concepción individualista de los actos, con el fin de poner más énfasis en su carácter teatral e iterativo: "la pertenencia a un sexo es un acto ya ensayado, es simultáneamente una reescenificación y una nueva experiencia de significaciones socialmente ya introducidas; es una forma cotidiana y ritualizada de su legitimación "(p. 312).La condición teatral e iterativa de nuestras identidades de género o identidades gener(iz)adas realza el momento y el poder de la incertidumbre que puede expresar nuestro cuerpo como actor y actuado. Puede seguir legitimando los rituales existentes y repetirlos o puede darse cuenta activamente de la "nueva experiencia de significaciones socialmente ya introducidas" (p. 312)y empezar a manipularlas en forma de iteraciones.

Existe una valoración tradicional de la belleza como "amenazante", porque puede ser "un peligro en tanto puede vencer la voluntad "Vélez (s.f.). Este temor lleva a que muchas mujeres bonitas según el canon cultural, se apoden como "bobas". Una estudiante de Klasse 11 en 2009, identificada por sus compañeros como "bonita" y "boba" por ejemplo, muestra en clase un comportamiento exagerado con comentarios precipitados que ella misma parece disfrutar mientras echa atrás su pelo largo cada 10 segundos y al mismo tiempo desarrolla por escrito trabajos muy bien pensados. Después de una conversación con ella en privado, comienza a participar de una manera muy constructiva en clase, con ideas muy bien pensadas y presentadas, a veces enmarcadas en comentarios de incredulidad de sus compañeros frenados por la profesora en el mismo instante. Pasan algunas semanas y ella vuelve a asumir su actuación de "boba", porque considera el cambio algo "difícil", probablemente también porque algunos profesores con los que hablo sobre ella, la consideran " $b o b a$ y bonita" o sea ¿es un peligro que pudiera vencer su voluntad, por lo que aquietan antes la voluntad de la potencialmente peligrosa? En total, el concepto incierto de la belleza no es limitado al cuerpo de la mujer, pues las estudiantes del grado $10^{\circ}$ en 2006 perciben que las y los profesores muestran preferencias por los hombres. Afirman que "las mujeres son menos atendidas y preparadas, ya que la mayoría de los entrenadores dicen que en las mujeres hay menos competencia y rivalidad, ya que piensan que son más débiles, perezosas y comprometidas con lo que hacen" (María grado $10^{\circ}$, sobre el tema Derechos de género 2006). Además, "a veces podemos percibir que algunas profesoras 
tienen ciertas preferencias con algunos alumnos, especialmente por ser lindo fisicamente" (Tati grado $10^{\circ}$, sobre el tema Derechos de género 2006).

La riqueza de las incertidumbres que se ejecutan desde y sobre las puestas en escena corporales en el aula como ilustrado en esos ejemplos, debe aprovecharse y transformarse de una forma coconstructiva y co-constructora para sacar a la luz del día cada vez más aspectos del currículo oculto. Una técnica prometedora para lograrlo es el juego dramático. Según las retroalimentaciones con estudiantes, éste "nos saca de lo académico", ayuda a combatir el aburrimiento y embrutecimiento. El juego de "Estatuas" por ejemplo, ilustra relaciones interpersonales de los presentes o provenientes de obras o realidades abordadas en la clase y requieren menos tiempo de preparación que los "juegos dramáticos". Estos equivalen a pequeñas obras teatrales que duran de tres a siete minutos. Como esta actividad requiere la disposición de todos los sentidos, también moviliza más diversión entre las y los participantes y al mismo tiempo exige la atención del o la facilitador(a), pues el desarrollo incierto del proceso creativo puede llevar a situaciones que oscilan entre lo cómico y lo revelador de asuntos muy, muy personales. Por eso es conveniente que el o la profesor/a se informe con anterioridad sobre estos aspectos, especialmente sobre una forma especial de juego dramático que es el "drama social". Citamos el discurso de un representante del partido "TRANS" que se realiza después de las presentaciones de los partidos "INTER" y "MULTI". El objetivo de esta actividad en un contexto político (partidos) previsto por la profesora consiste en discutir y cuestionar con los estudiantes de 16 ó 17 años los tres conceptos y sus efectos en las sociedades en las que conviven varios y distintos grupos sociales y étnicos. La presentación en cambio, se concentra primero en la TRANS-ición de la sección Primaria a la sección Secundaria y los efectos que experimentan en esa fase las y los estudiantes. Después profundiza en las TRANS-iciones en la vida familiar:

También se sufren cambios en la familia cuando por ejemplo en algunas familias se separan los papás, entonces hay cambio para los... pues, para uno, o cuando se cambian de casa o de ciudad porque al papá lo cambiaron de empleo; listo; entonces es la forma de uno adaptarse a lo que le toca. También existen cambios con los amigos, el... pues, con las amistades, uno también no siempre va a ser amigo de alguien y no lo va a tratar igual, sino que puede también tener una pelea o cambiar de ambiente y tiene que adaptarse a otros nuevos amigos. MacKeldey, (2010a).

En un primer momento puede surgir la incertidumbre, si este discurso refleja una comprensión de la actividad, y al mismo tiempo puede surgir la claridad, que son justamente estos aspectos veleidosos de instrucciones abiertas que incluyen más a las y los estudiantes como actores de conocimiento. La puesta en escena discursiva y corporal realizada por el Partido TRANS refleja en todos los sentidos la exigencia de volver político lo personal y puede ser el ancla para nuevas actividades que abarcan por ejemplo, el contexto socio-histórico en el que surgió esta exigencia o las implicaciones de la exigencia para la vida política (local, regional, nacional, internacional). Ello siempre respetando las modificaciones que pueden ofrecer las siguientes puestas en escena discursivas y corporales de las y los estudiantes. El primer marco de referencia para las y los estudiantes suele ser la vida cotidiana y para poder abarcar el valor de este campo de experiencia desde una perspectiva co-constructiva, puede considerarse el método documental.

\section{Hacer malabarismos con incertidumbres contingentes: el método documental}

Para hacer más asequibles los juegos identitarios y otras incertidumbres de convivencia, el método documental desarrollado por Bohnsack (2003) se centra en una cotidianidad visceral de las personas, es decir, en "las condiciones en las que se producen la seguridad existencial, la integración colectiva y el entendimiento inmediato. Esta realidad existencial de las reservas de saberes implícitos que orientan los actos, está anclada a más profundidad que la realidad de las instituciones, roles y normas descritas en la obra de Berger y Luckmann" (p. 231).

El método documental compara prácticas conjuntivas, es decir, pone en relación escenificaciones corporales y discursivas provenientes de contextos afines, para alcanzar un determinado fundamento empírico para las cotidianidades o "realidades" puestas en escena. Un escenario muy diciente 
para la aplicación del método documental y la detección de cotidianidades o realidades viscerales es el siguiente: los integrantes de un grupo de estudiantes de grado $10^{\circ}$ en 2005 se sientan en forma de círculo y empiezan a co-construir discursivamente vidas de jóvenes afines a su campo de experiencia. La palabra se entrega con ayuda de una pelotica- una técnica que debe aplicarse sólo en grupos conocidos ya que la incertidumbre de recibir o no la palabra puede causar estrés en algunos participantes-. La actividad descrita se ve enriquecida cuando un integrante plasma en caricaturas los polos entre los cuales oscilan las identidades co-construidas e ilustra unos fragmentos del juego identitario realizado por los jóvenes que deberían considerar los educadores en el aula.

Figura 4: Caricatura ilustrativa del juego identitario, realizada por un estudiante participante Daniel Tamayo Arcilla

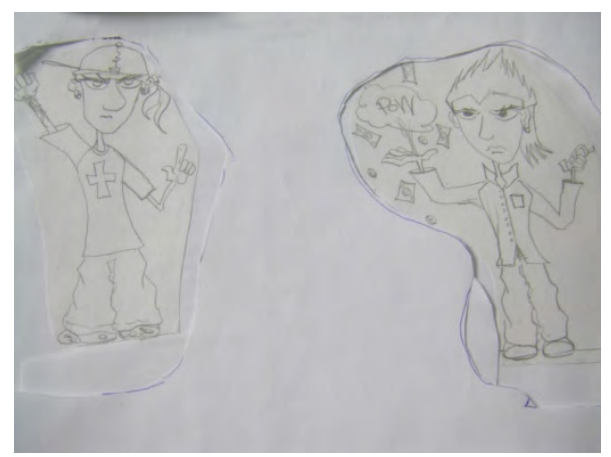

Fuente: portada del cuadernillo "Performing Transcultural Gender (2005)”, documento inédito.

\section{Aprovechar la retroalimentación como incertidumbre}

Cada una de las técnicas mayeútico-didácticas presentadas que forman parte del repertorio de la PPI debe ir acompañada al final por una evaluación y/o retroalimentación. Ambas son un aspecto indispensable de los procesos educativos y deben realizarse con regularidad de forma oral aproximadamente cada semana y por escrito cada bimestre. También las y los docentes de la educación superior deben considerar formas más personalizadas que las encuestas estandarizadas para poder sondear las ventajas y desventajas de los procesos de enseñanza y aprendizaje. En los comienzos, la evaluación puede provocar incertidumbres en el educador ya que los y las estudiantes suelen ser muy sinceros. Al mismo tiempo, la retroalimentación es una gran oportunidad para la autorreflexión y la co-construcción de los saberes discursivos y corporales.

En lo siguiente, presentamos los dos polos de las retroalimentaciones oscilantes de un $11 \mathrm{er}$ grado para el primer bimestre de 2014. Se aclara el meta-objetivo de este bimestre: preparación para el análisis de textos según los criterios IB, la intención de las micro-secuencias se deja a propósito en el aire. Por un lado, porque las clases siempre procuran una mayor apertura hacia la co-construcción posible y segundo, se formulan a propósito las instrucciones con frecuencia de una manera extremamente abierta. En las reflexiones sobre las instrucciones abiertas, los estudiantes expresan su confusión sobre el papel activo del estudiante; surgen comentarios como hay "demasiado ruido", hay que "preguntar a compañeros" o "buscar ideas que podría ser la meta". Se despiertan determinadas incertidumbres en la profesora, cuando más de un(a) estudiante de 17 ó 18 años considera difícil que "uno debe pensar qué es lo que el profesor quería". ¿Qué procesos de enseñanza y aprendizaje en una institución educativa, que pretende formar sujetos autónomos, llevan a que muchos estudiantes suponen el aprendizaje no como un valor personal y enriquecedor para el proyecto de vida, sino como hacer "lo que el profesor quería"? Pero existen al mismo tiempo comentarios positivos, aunque en número reducido, acerca de que las instrucciones no claras "permiten jugar más con la creatividad" y "se logran ver las distintas interpretaciones de los estudiantes". 


\section{Una cierta conclusión co-construida sobre la incertidumbre en la educación}

En la fase de la retroalimentación, una estudiante interpreta las instrucciones de una forma muy creativa y eterniza su comentario en una camiseta. La aprovechamos para dar fin a la presente propuesta de considerar en la educación las incertidumbres inherentes al siglo XXI y a nuestro ser sujetos relacionales con la ayuda de la pedagogía performativa intercultural. Sus métodos para o sus caminos hacia la co-construcción de sujetos que se (auto)entiendan y representen como actores actuados, procuran formar una mayor conciencia sobre las múltiples identidades simultáneas y sobre la responsabilidad que surge con la percepción de la horizontalidad de las interrelaciones humanas, en las que nunca podemos estar seguros que el otro entienda o interprete análogamente nuestras puestas en escena corporales o discursivas.

Figura 5: Camiseta estampada por la estudiante Susana Carrizosa Botero como ejercicio de retroalimentación

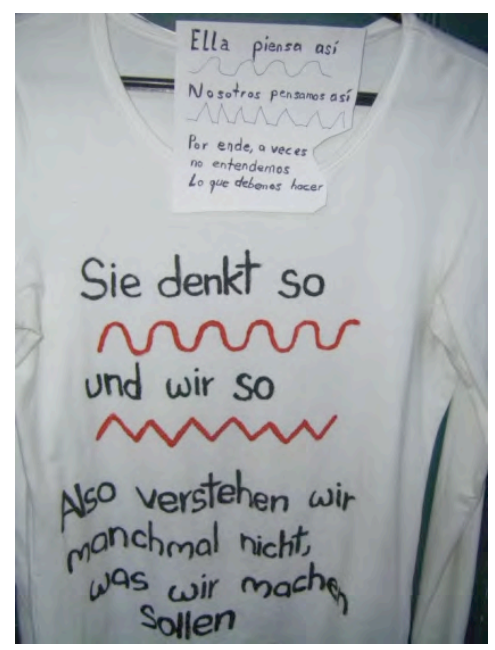

Fuente: Foto tomada por la autora, 2014. 


\section{REFERENCIAS}

Bohnsack, R. (2001). Typenbildung, Generalisierung und komparative Analyse. Grundprinzipien der dokumentarischen Methode. En R. Bohnsack et al. (ed.), Die dokumentarische Methode und ihre Forschungspraxis. Grundlagen qualitativer Sozialforschung (pp. 225-252). Opladen: Leske und Budrich.

Borrajo, G. (2012). Miguel Ángel Escotet: Educar para la incertidumbre es absolutamente necesario en estos tiempos. Revista Eduga, 59. Recuperado el 20 de diciembre de 2013 desde: http://www.miguelescotet.com/webnews/edugainterviewES.html

Brinkmann, M. (2009). Fit für Pisa? - Bildungsstandards und performative Effekte im Testregime. Vorschläge zur theoretischen und praktischen Differenzierung von Bildungsforschung und Aufgabenkultur. En Bilstein et al.(ed.),Standardisierung - Kanonisierung. Erziehungswissenschaftliche Reflexionen.(pp.97-106). Wiesbaden: Schriftenreihe der Kommission Allgemeine Erziehungswissenschaft der DGFE. Recuperado el 10 de septiembre de 2013 desde http://download.e-bookshelf.de/download/0000/0173/59/L-G-0000017359-0002372499.pdf

Butler, J.(2002). Performative Akte und Geschlechterkonstitution. Phänomenologie und feministische Theorie. En U. Wirth (ed.), Performanz. Zwischen Sprachphilosophie und Kulturwissenschaften (pp. 301-320). Frankfurt del Meno, Alemania: Suhrkamp.

Dyson, M. (2007). My Story in a Profession of Stories: Auto-Ethnography - an Empowering Methodology for Educators. Australian Journal of Teacher Education, 32(1), pp. 36-48.

Freire, P. (1973). Pädagogik der Unterdrückten. Stuttgart, Berlín: Editorial Kreuz.

Garzón Martínez, M. T. (2007). Proyectos Corporales. Errores subversivos: hacia una performatividad decolonial del silencio. Nómadas, 26, pp. 154-165.

Gómez, R. del S.; González, J. (2003). Design: Designar/Diseñar el Cuerpo Joven y Urbano. Un estudio sobre la cultura somática de jóvenes integrados en Cali. Cali, Colombia: Anzuelo Ético.

Hall, S. y Gay, P. (ed.) (2003).Cuestiones de identidad cultural. Buenos Aires, Argentina: Amorrortu.

Kristeva, J. (1997). Strangers to Ourselves. New York: Columbia University Press.

Kusanowsky, K. (2013). Leben mit der Paranoia: Into the wild - re:publica 2014. Differentia. Recuperado el $1^{\circ}$ de junio de 2014 desde http://differentia.wordpress.com/2013/12/16/lebenmit-der-paranoia-into-the-wild-republica-2014/

Lotter, W. [@WolfLotter]. (s.f.).Lo salvajey el conocimiento son lo mismo.

MacKeldey, A. M. (2010a). El Arte de aprender a nadar entre dos aguas-Die Kunst zu lernen, wie man zwischen zwei Stühlen sitzt (Tesis doctoral). Medellín: Universidad de Antoquia.

- (2010b). Entrevista con Luiyima. Envigado: documento inédito.

Morin, E. (1999). Siete saberes necesarios para la educación del futuro. Paris: UNESCO. Recuperado el 10 de mayo de 2014 desdehttp://unesdoc.unesco.org/images/0011/001177/117740so.pdf

Muth, C. (s.f.). Dialogische Pädagogik: Identitätsbildung durch die Andersheit. Recuperado el 20 de mayo de 2010 desde www.corneliamuth.de/pdfs/habilitationskonzept-muth.pdf

Notas en rojo para la educación en Colombia. (2013). [Revista Semana] Recuperado el 8 de febrero de 2014 desde http://www.semana.com/nacion/articulo/bajos-resultados-de-la-educacioncolombiana-en-2013/368197-3

Olave-Arias, G., Rojas-García, I., Cisneros-Estupiñán, M. (2013). Deserción universitaria y alfabetización académica. Educación y educadores, 16(3), pp. 455-471.

Parra Sandoval, R. (1997). El tiempo mestizo: escuela y modernidad en Colombia. En Proyecto Atlántida. Estudio sobre adolescentes escolares en Colombia. Tomo I: La Cultura Fracturada. Ensayos sobre la adolescencia colombiana. Bogotá, Colombia: Fundación FesColciencias-TM.

PONS - Wörterbuch für Schule und Studium. (1999). Stuttgart, Düsseldorf,Leipzig: Klett.

Republica (2014). Call for papers - Werde Teil der Republica 2014.Recuperado el 4 de marzo de 2014 desde http://re-publica.de/news/call-papers-werde-teil-republica-2014

Vélez, B. (Inédito). Espectáculo corporal ¿Frontera de violencias insidiosas de género? 
Wang, H. (2005). Aporias, Responsibility and the Im/possibility of Teaching Multicultural Education. Educational Theory, 55(1), pp. 45-60.

Wulf, C. (2007). Pädagogik des Performativen. Weinheim y Basel: Beltz.

Zirfas, J. (2001). Dem Anderen gerecht werden. Das Performative und die Dekonstruktion bei Jacques Derrida. En C. Wulf et al. (ed.): Grundlagen des Performativen. Eine Einführung in die Zusammenhänge von Sprache, Macht und Handeln. Weinheim y Munich: Juventa.

\section{SOBRE LA AUTORA}

Anja María MacKeldey: Doctora en Educación: Investigadora asociada al grupo de investigación Unipluriversidad-Universidad de Antioquia Sede Medellín; Docente Alemán como Lengua Extranjera (Secundaria y GIB) Colegio Alemán Medellín. 